\title{
Immunochemical Characterization of Neisseria meningitidis Serotype Antigens by Immunodiffusion and SDS-Polyacrylamide Gel Electrophoresis Immunoperoxidase Techniques and the Distribution of Serotypes Among Cases and Carriers
}

\author{
By J. T. POOLMAN, * C. T. P. HOPMAN AND H. C. ZANEN \\ Laboratory of Hygiene, University of Amsterdam, Mauritskade 57, \\ 1092 AD Amsterdam, The Netherlands
}

(Received 23 March 1979; revised 2 July 1979)

\begin{abstract}
The chemical nature of the antigens of the meningococcal serotypes described by Frasch and colleagues was determined by a combination of immunodiffusion and the SDS-polyacrylamide gel electrophoresis immunoperoxidase technique (SGIP). It was confirmed that the serotype antigens of the outer membrane of serotypes $1,2,6,9,11$ and 12 were proteins, whilst those of serotypes 4, 5 and 8 were lipopolysaccharides. Serotype 2 can now be divided into three related types, provisionally called $2 \mathrm{a}$ (originally serotype 2 ), $2 \mathrm{~b}$ and $2 \mathrm{c}$ with the specific antigens being proteins having molecular weights of 41000,41500 and 41500 , respectively. A total of 195 strains of meningococci isolated from patients and carriers in the Netherlands and 20 serogroup $\mathrm{Y}$ strains from patients in the U.S.A. were serotyped by means of immunodiffusion. Serotype $2 \mathrm{a}$ could be demonstrated in some strains belonging to the serogroups B (only those from carriers), C, W-135 and Y (only those from the U.S.A.). The W-135 strains isolated from patients in this series more often belonged to serotype $2 a$ than did the W-135 strains from carriers. Serotype $2 b$ was present in about half of the serogroup B and a few serogroup C strains isolated from patients with meningitis, but absent in serogroup B and C strains from carriers. Serotype $2 c$ could only be demonstrated in serogroup Y strains, both from the Netherlands and the U.S.A. The other serotypes were found only sporadically.
\end{abstract}

\section{INTRODUCTION}

Neisseria meningitidis has been divided into nine serogroups $\left[\mathrm{A}, \mathrm{B}, \mathrm{C}, \mathrm{D}, \mathrm{X}, \mathrm{Y}, \mathrm{Z}, \mathrm{Z}^{1}\right.$ (29E) and W-135] with antisera raised to the capsular polysaccharides (Branham, 1953; Slaterus, 1961; Evans et al., 1968). The chemical structure of most of the group-specific polysaccharides has been elucidated (Jennings et al., 1977; Bhattacharjee et al., 1978). Further classification into serotypes is based on non-capsular antigens present in the outer membrane (Gold \& Wyle, 1970; Frasch \& Chapman, 1972a). Analysis of some of these serotype antigens by Frasch and Zollinger has shown them to be either proteins or lipopolysaccharides (Zollinger et al., 1972, 1974; Frasch \& Gotschlich, 1974; Frasch et al., 1976; Zollinger \& Mandrell, 1977).

To identify an organism accurately, to study the epidemiology and pathogenesis of the infection to which it gives rise and to develop an effective vaccine against that infection may depend upon knowledge of the detailed antigenic structure of the species. In addition, antisera specific for particular antigens are required to allow reliance to be placed upon immunological methods of identification such as immunodiffusion, serum bactericidal activity 
and agglutination (Gold \& Wyle, 1970; Frasch \& Chapman, 1972 a, b; Jones \& Tobin, 1976; Mandrell \& Zollinger, 1977). It was to fulfil these requirements that the recently developed SDS-polyacrylamide gel electrophoresis immunoperoxidase technique (SGIP) of $v$. Raamsdonk et al. (1977) was applied to the analysis of the outer membranes of the meningococcus, the results of which form the basis of this communication. In addition, the distribution of the various meningococcal serotypes amongst a group of strains isolated from both patients and carriers is reported.

\section{METHODS}

Bacterial strains. The prototype meningococcal strains M1080 (serotype 1), B16B6 (serotype 2), M986 (serotype 2, 7), M1011 (serotype 2, 10), M981 (serotype 4), M992 (serotype 5), M990 (serotype 6), M978 (serotype 8), M982 (serotype 9), M136 (serotype 11) and S3032 (serotype 12) were obtained from Dr C. E. Frasch (Bureau of Biologics, Food and Drug Administration, Bethesda, Md, U.S.A.). These were used to study the nature of the antigen(s) in the various serotypes and to prepare type-specific antisera. The serotypes 3, 7 and 10 were not included in the series because the original prototype of serotype 3 was unsuitable for use and the antigens of serotypes 7 and 10 , whenever present, occurred in conjunction with serotype 2 (Frasch \& Chapman, $1972 a, b$ ). In addition, a total of 215 strains of $N$. meningitidis, 130 from cases of meningococcal disease and 85 from healthy carriers, were collected and examined with respect to their serogroup and serotype. With the exception of 20 serogroup $Y$ strains isolated from cases of meningococcal disease and obtained from $\mathrm{Dr} \mathrm{H}$. A. Feldman (State University of New York, Upstate Medical Centre, Syracuse, N.Y., U.S.A.), every strain was isolated in the Netherlands from the blood, cerebrospinal fluid or nasopharynx of patients or carriers. The four strains studied in detail -2996, 3006 (both serogroup B, serotype 2b), 2396 and 3459 (neither groupable, serotype $2 \mathrm{c}$ ) - were isolated in the Netherlands from patients with meningitis.

Cultural conditions and isolation of outer membrane complexes. Organisms were cultured in Trypticase Soy Broth (Difco) and their outer membrane complexes (OMC) were isolated from overnight cultures as described by Poolman et al. (1978).

Separation and chemical identification of OMC. SDS-polyacrylamide gel electrophoretic (SDS-PAGE) separation of the components of OMC was carried out as described by Poolman et al., (1978). The following proteins (Boehringer) were used as molecular weight standards: cytochrome $c$ (mol. wt 12500); chymotrypsinogen A (mol. wt 25000); aldolase (mol. wt 39500, polypeptide chains); ovalbumin (mol. wt 45000); catalase (mol. wt 60000, polypeptide chains); bovine albumin (mol. wt 67000).

Gel immunoperoxidase technique (GIP). After SDS-PAGE, the separated OMC were analysed immunologically by the gel immunoperoxidase technique of v. Raamsdonk et al. (1977), incorporating a few modifications. The combination of SDS-PAGE and GIP has been called SGIP (SDS-polyacrylamide gel electrophoresis immunoperoxidase technique). The SDS gels were placed on metal holders covered with chromatographic paper, frozen in liquid nitrogen (keeping the metal holder in and the gel above the liquid nitrogen) and sliced longitudinally at $-35^{\circ} \mathrm{C}$ with a cryostat microtome (Bright 5030$)$ into $50 \mu \mathrm{m}$ slices $(5 \times 5 \mathrm{~cm})$. The slices were freed from SDS and stored at $-20^{\circ} \mathrm{C}$ in a mixture of ethanol/acetic acid $(6: 1, \mathrm{v} / \mathrm{v})$. Before use, the slices were washed in PBS ( $2 \mathrm{~mm}-\mathrm{NaH}_{2} \mathrm{PO}_{4}, 8 \mathrm{mM}-\mathrm{Na}_{2} \mathrm{HPO}_{4}$ and $0 \cdot 14 \mathrm{M}-\mathrm{NaCl} \mathrm{pH}$ 7.2) to remove the ethanol/acetic acid mixture. The slices were spread and incubated on glass slides $(10 \times 10 \mathrm{~cm})$ with rabbit antisera against meningococci (dilution series $1: 2$ to $1: 25, \pm 15 \mu 1$ per cm${ }^{2}$ gel) in a moist atmosphere at room temperature for $30 \mathrm{~min}$ and then at $4{ }^{\circ} \mathrm{C}$ overnight. After washing with PBS for $1 \mathrm{~h}$ the slices were incubated with goat anti-rabbit-IgG-peroxidase-labelled antiserum (Miles Laboratories, Elkhart, Ind., U.S.A.), specific for both light and heavy chains of rabbit IgG, (dilution $1: 25$ ) at room temperature for $2 \mathrm{~h}$. Finally, the slices were washed with PBS at room temperature for $1 \mathrm{~h}$ and stained with 3-amino-9-ethylcarbazole (Sigma) for 15 to $30 \mathrm{~min}$. The staining solution contained $0.05 \mathrm{M}$-acetate, $0.01 \%$ (v/v) $\mathrm{H}_{2} \mathrm{O}_{2}, 5 \%$ $N, N$-dimethylformamide and $0.04 \%$ (w/v) 3-amino-9-ethylcarbazole $\mathrm{pH} 5 \cdot 0$. The staining solution was removed from the slices by washing with water. In turning red, the reacting antigens became visible. Control reactions were performed with goat anti-rabbit-IgG-peroxidase alone and in combination with pre-immunization rabbit serum.

Micro-precipitation. Ouchterlony immunoprecipitation was carried out on glass slides $(5 \times 5 \mathrm{~cm})$ which were layered with $1.5 \%(\mathrm{w} / \mathrm{v})$ agar Noble (Difco) in water. For serogrouping, immunodiffusion was carried out as described by Slaterus (1961). For serotyping, the antiserum wells were filled $6 \mathrm{~h}$ after the antigen wells; both the antigen and antiserum wells were filled twice. In all cases purified $\mathrm{OMC}$ were used as antigen. The slides were incubated in a moist atmosphere at room temperature for 24 to $48 \mathrm{~h}$. After washing in PBS the slides were dried and stained with Coomassie brilliant blue $(0 \cdot 5 \%$, w/v) in acetic acid/water/ethanol $(1: 4 \cdot 5: 4 \cdot 5$, by vol. $)$ for $5 \mathrm{~min}$. 
Antisera. Antisera against serogroups were prepared according to Slaterus (1961) using the reference strains previously described (Poolman et al., 1978). Antisera to a serotype 1 strain (M266) and the prototype strains B16B6 (serotype 2), M1011 (serotype 2, 10), M992 (serotype 5), M990 (serotype 6) and M982 (serotype 9) were kindly supplied by Dr C. E. Frasch. In addition, antisera to the prototype strains obtained from $\mathrm{Dr}$ C. E. Frasch were raised in 2 month-old cross-bred white $N^{\top}$ ew Zealand rabbits (approx. $2 \cdot 5 \mathrm{~kg}$ wt) according to the immunization schedule of Frasch \& Chapman (1972a). When satisfactory precipitating titres were reached, the rabbits were bled by cardiac puncture. To obtain highly specific antisera, the serum from each immunized rabbit was then absorbed with an equivalent volume of packed meningococci to remove crossreacting antibodies. After absorption at $37^{\circ} \mathrm{C}$ for $4 \mathrm{~h}$ and then at $4{ }^{\circ} \mathrm{C}$ overnight, the bacteria were removed by centrifuging at $20000 \mathrm{~g}$ for $20 \mathrm{~min}$ at $4{ }^{\circ} \mathrm{C}$. The following absorptions were found to be necessary: antiserotype 1 with meningococci M992 and M978; anti-2a with M136, 2996 and 2396; anti-2b with M1011 and 2396; anti-2c with M.1011 and 2996; anti-4 with M1011, M992 and S3032; anti-5 with M978 and M136; anti-6 with M1011, M136 and S3032; anti-8 with M981 and M992; anti-9 with M1080, M1011 and M136; anti-11 with M1011, M992 and M990; anti-12 with M981, M992 and M982. Before use the specificity of each absorbed antiserum was verified by Ouchterlony immunoprecipitation tests against each of the serotypes. Goat anti-rabbit-IgG-peroxidase-labelled antiserum was absorbed with a mixture of all prototype meningococci to remove any reactivity against the OMC. The antisera used in the experiments reported were prepared in our laboratory, unless stated otherwise.

\section{RESULTS}

\section{Immunochemistry of serotype antigens, other than serotype 2}

Outer membrane complexes from prototype strains $1,4,5,6,8,9,11$ and 12 were analysed on two parallel gel slabs by SDS-PAGE. The remainder of these gels after longitudinal slicing were stained for proteins or lipopolysaccharides. The major outer membrane proteins (OMP) were located in the middle of the gel slab, indicative of a molecular weight between 25000 and 50000 (Fig. 1 $a$ ), and the lipopolysaccharides were located at the anodic end (Fig. $1 b$ ). The position of the capsular polysaccharides, shown using group-specific antisera, was at the cathodic end of the gel slab (Poolman et al., 1978).

The treatment of the gel slices with any one of the specific serotype antisera, as judged by immunodiffusion, followed by development with GIP and staining with 3-amino-9-ethylcarbazole revealed extensive cross-reactivity (Fig. $1 c, e, g$ ). This cross-reactivity occurred not only with our own specific serotype antisera but also with those provided by Dr C. E. Frasch. However, further absorption with heterologous prototy pe serotype strains rendered the antisera specific as measured by the GIP technique (Fig. $1 d, f, h$ ). The specific antigens of serotypes 4, 5, 6, and 11 were identified as single entities by this procedure. However, despite repeated absorption of the antisera to serotypes $1,8,9$ and 12 with heterologous prototype serotype strains, more than one antigen remained as revealed by the GIP technique (Fig. 1 h). Further identification of the principal antigen responsible for type specificity in serotypes 1, 8 and 12 was obtained when these prototype serotypes were compared with strains isolated from patients and/or carriers and classified as of the same type by immunodiffusion yet different from each other with respect to their protein patterns. An example of this approach is given in Fig. 2 showing that the principal antigen responsible for specificity in serotype 12 is the protein of molecular weight 27000 rather than the additional one in the prototype serotype 12 whose molecular weight is 45000 . Following this approach, the chemical nature of the only or principal antigen of the several serotypes, with the exception of serotype 9, was determined. Absolute characterization of the principal antigen of serotype 9 was not possible because no strain(s) immunologically identical to this prototype serotype was isolated from either patients or carriers.

The detailed chemical nature of the serotype antigens is given in Table 1. 


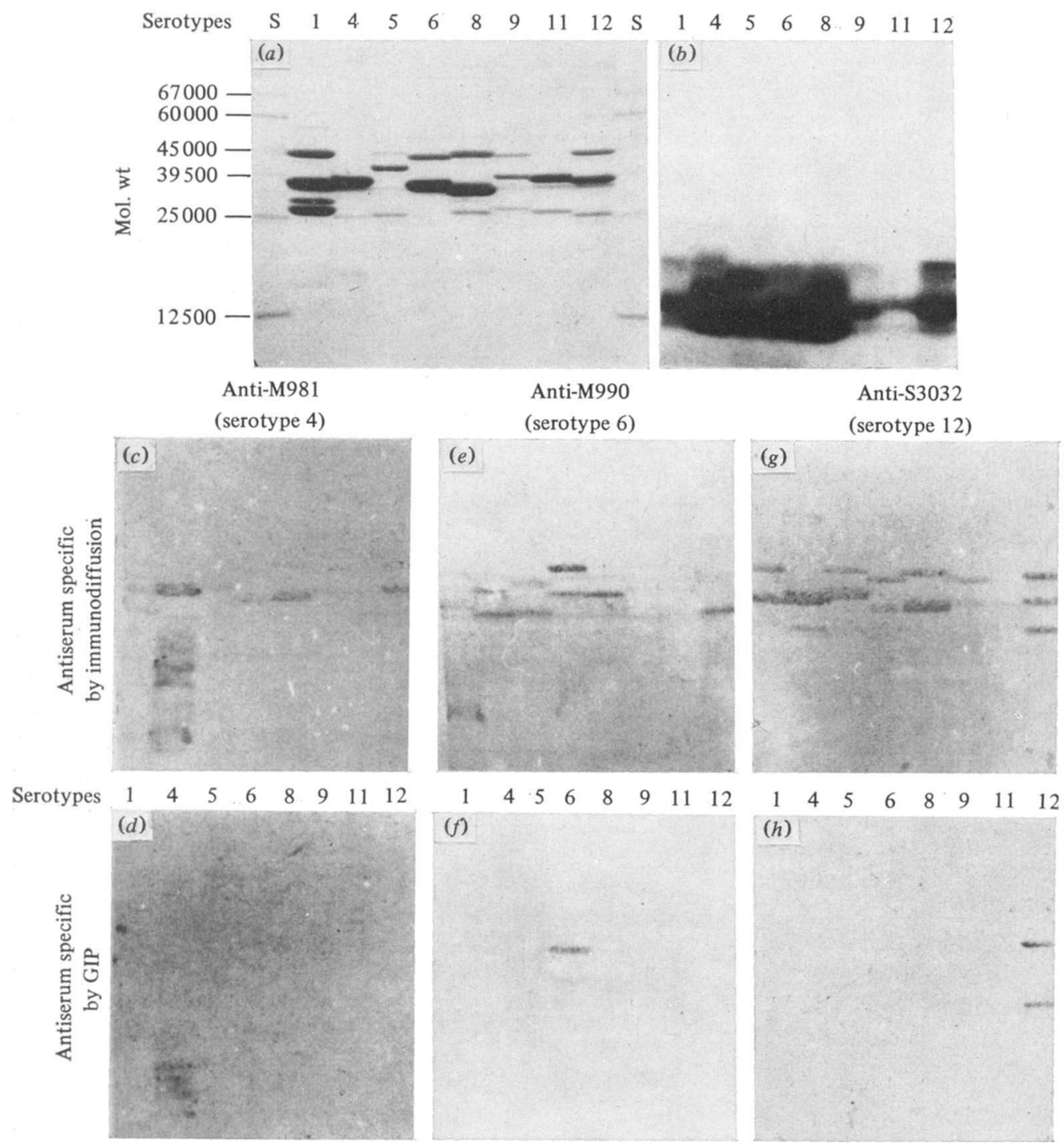

F ig 1. Identification of the type-specific antigens of prototype serotype strains of $N$. meningitidis by SDSं-PAGE and development with (a) Coomassie brilliant blue, $(b)$ Schiff's reagent or $(c$ to $h)$ rabbit antisera to serotypes 4, 6 and 12 and goat anti-rabbit-IgG-peroxidase-labelled antiserum (GIP). (S indicates protein standards.)

\section{Immunochemistry of serotypes $2 \mathrm{a}, 2 \mathrm{~b}$ and $2 \mathrm{c}$}

Immunodiffusion failed to differentiate between the prototype serotype 2 strains B16B6 (serotype 2), M986 (serotype 2, 7) and M1011 (serotype 2, 10). We regarded these strains as belonging to one serotype, namely serotype 2, thereby following the approach of Frasch \& Friedman (1977). Antisera raised against the prototype serotype 2 strains B16B6 and M1011, which after thorough absorption did not react with heterologous prototype strains, reacted with many strains of meningococci isolated in the Netherlands when tested by immunodiffusion. Some of these reacting strains showed immunological identity with the prototype serotype 2 strains, while others did not (Fig. 3). By raising antisera against the strains involved, these immunological differences could be characterized. After absorption, three different type-specific antisera, as determined by immunodiffusion, emerged (Fig. 3). The 


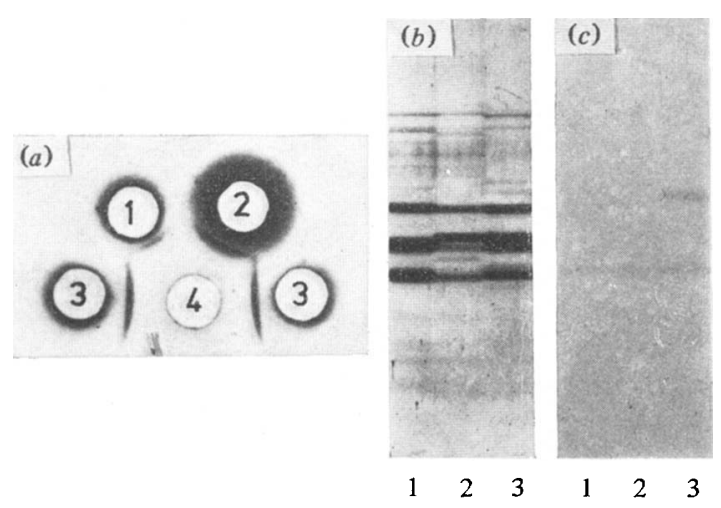

Fig. 2. Analysis of two freshly isolated serotype 12 strains of $N$. meningitidis [the serogroup B carrier strain 3206 (1) and the serogroup C patient strain 3242 (2)] and the prototype serotype 12 strain S3032 (3) by (a) immunodiffusion against rabbit anti-S3032 (4) serum, and by SDS-PAGE developed with $(b)$ Coomassie brilliant blue and $(c)$ rabbit anti-S3032 serum and goat anti-rabbit-IgG-peroxidase-labelled antiserum (GIP).

Table 1. Chemical nature and molecular weight of the principal antigens of N. meningitidis serotypes

\begin{tabular}{ccc} 
Serotype & \multicolumn{2}{c}{ Antigen } \\
\cline { 2 - 3 } 1 & Nature & $10^{-3} \times$ Mol. wt \\
4 & P & 36 \\
5 & LPS & ND \\
6 & LPS & ND \\
8 & P & 43 \\
9 & LPS & ND \\
11 & P & 28 and /or 44 \\
12 & P & 37 \\
$2 \mathrm{a}$ & $\mathrm{P}$ & 27 \\
$2 \mathrm{~b}$ & $\mathrm{P}$ & 41 \\
$2 \mathrm{c}$ & $\mathrm{P}$ & 41.5 \\
& $\mathrm{P}$ & 41.5
\end{tabular}

P, Protein; LPS, lipopolysaccharide; ND, not determined.

serotypes identified with these antisera are provisionally designated serotype 2a (Frasch serotype 2, strains B16B6 and M1011), 2b (strains 2996 and 3006) and 2c (strains 2396 and 3459).

Incubation of OMC from these six strains separated by SDS-PAGE with antisera specific for serotypes $2 \mathrm{a}, 2 \mathrm{~b}$ and $2 \mathrm{c}$ followed by treatment with GIP and staining revealed the OMP antigen(s) of the three serotypes to be distinct entities (Fig. $4 b, c, d$ ); all were proteins (Fig. 4a).

Exposure of OMC from the prototype serotype 2 strains B16B6 and M1011 to typespecific serotype 2 antiserum, produced in our own laboratory or received from Dr C. E. Frasch, showed them to possess two common but type-specific OMP antigens of molecular weights 41000 and 43500 . However, exposure of the same two serotype OMC to anti-M1011 revealed the presence of a third OMP antigen of molecular weight 28000 in M1011 (Fig. $4 b$ ). This additional antigen probably represents the serotype 10 antigen. The analysis of OMC from freshly isolated strains of meningococci, identical on immunodiffusion to these two prototype strains, provisionally designated serotype $2 \mathrm{a}$, showed the OMP of molecular weight 41000 to be the serotype $2 \mathrm{a}$ antigen.

Separation and immunological analysis of OMC from strains 2996 and 3006, designated serotype $2 \mathrm{~b}$, showed them to possess a common but type-specific OMP antigen of molecular 


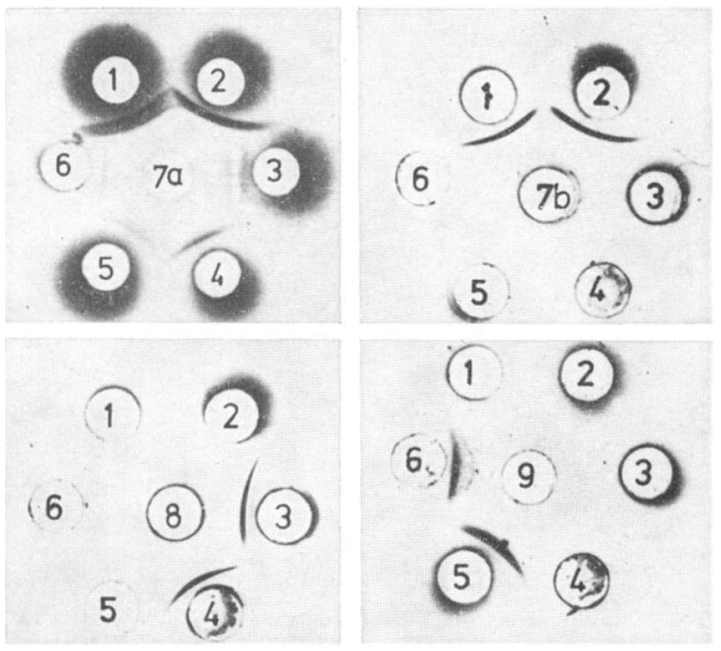

Fig. 3. Immunodiffusion reactions between prototype serotype 2 strains of $N$. meningitidis [B16B6 (1) and M1011 (2)] and four freshly isolated strains of N. meningitidis [2996 (3), 3006 (4), 2396 (5) and 3459 (6) ] and rabbit anti-M1011 (7a), anti-M1011 absorbed with 2996 and 2396 (7b), anti-3006 (8) and anti-2396 (9) sera.

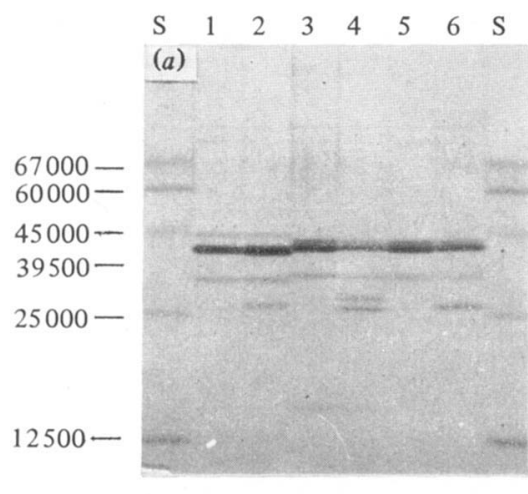

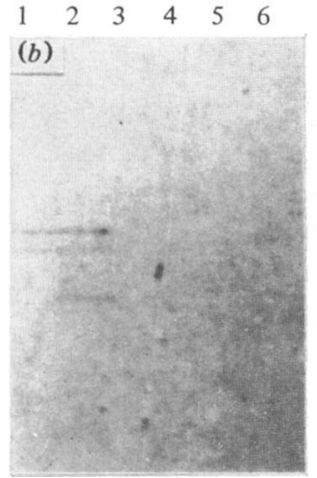

Anti-M1011

(serotype 2a)

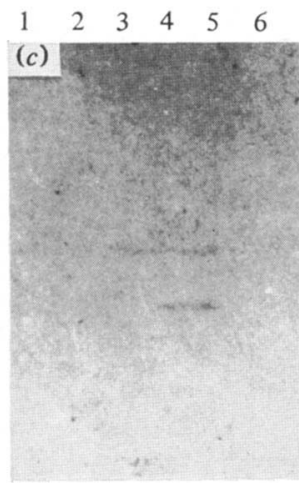

Anti-3006

(serotype 2b)

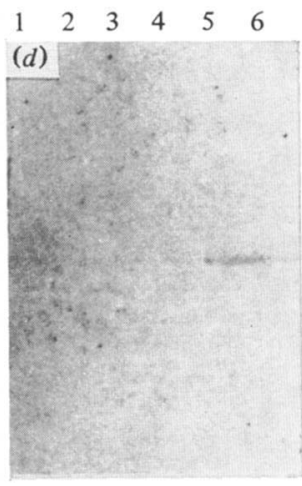

Anti-2396

(serotype 2c)

Fig. 4. Identification of the type-specific antigens of the related serotypes $2 \mathrm{a}, 2 \mathrm{~b}$ and $2 \mathrm{c}$ of N. meningitidis by SDS-PAGE and development with (a) Coomassie brilliant blue or $(b$ to $d$ ) rabbit antisera to serotypes $2 \mathrm{a}, 2 \mathrm{~b}$ and $2 \mathrm{c}$ and goat anti-rabbit-IgG-peroxidase-labelled antiserum(GIP). Test strains: serotype 2a, B16B6 (1) and M1011 (2); serotype 2b, 2996 (3) and 3006 (4); serotype 2c, 2396 (5) and 3459 (6). (S indicates protein standards.) 
Table 2. Distribution of serotypes among 215 strains of $N$. meningitidis

\begin{tabular}{|c|c|c|c|c|c|c|c|c|c|c|c|c|c|c|}
\hline \multirow[b]{2}{*}{ Source } & \multirow[b]{2}{*}{ Serogroup } & \multirow{2}{*}{$\begin{array}{l}\text { No. in } \\
\text { group }\end{array}$} & \multicolumn{12}{|c|}{ No. of each serotype } \\
\hline & & & 1 & 4 & 5 & 6 & 8 & 9 & 11 & 12 & $2 a$ & $2 b$ & $2 \mathrm{c}$ & NT \\
\hline \multirow[t]{7}{*}{ Patients } & A & 20 & - & - & - & - & 3 & - & - & - & - & - & - & 17 \\
\hline & B & 40 & 3 & - & - & - & 3 & - & 1 & - & - & 22 & - & 11 \\
\hline & $\mathrm{C}$ & 20 & 2 & - & - & - & 1 & - & 2 & 1 & 4 & 2 & - & 8 \\
\hline & W-135 & 20 & -- & - & - & - & - & - & - & - & 18 & - & - & 2 \\
\hline & Y (Neth.) & 10 & - & - & - & - & 1 & - & - & - & - & - & 3 & 6 \\
\hline & Y (U.S.A.) & 20 & -- & - & - & - & - & - & 一 & - & 5 & - & 10 & 5 \\
\hline & Total & 130 & 5 & - & - & - & 8 & - & 3 & 1 & 27 & 24 & 13 & 49 \\
\hline \multirow[t]{10}{*}{ Carriers } & $\mathrm{A}$ & 5 & - & - & - & - & - & - & - & - & - & - & - & 5 \\
\hline & B & 20 & - & - & - & 2 & 2 & - & 1 & - & 3 & - & - & 12 \\
\hline & $\mathrm{C}$ & 10 & - & - & - & - & - & - & - & - & 2 & - & - & 8 \\
\hline & $Z^{\prime}(29 E)$ & 10 & - & - & - & - & - & - & - & - & - & - & - & 10 \\
\hline & W-135 & 10 & -- & - & - & - & - & - & - & - & 3 & - & - & 7 \\
\hline & $\mathrm{x}$ & 10 & $\ldots$ & - & - & - & - & - & - & - & - & - & - & 10 \\
\hline & Y & 10 & - & - & - & - & - & - & - & - & - & - & 3 & 7 \\
\hline & NG & 10 & 1 & - & - & - & - & - & - & - & - & - & - & 9 \\
\hline & Total & 85 & 1 & - & - & 2 & 2 & - & 1 & - & 8 & - & 3 & 68 \\
\hline & Grand total & 215 & 6 & - & - & 2 & 10 & - & 4 & 1 & 35 & 24 & 16 & 117 \\
\hline
\end{tabular}

weight 41500 , with strain 3006 , as revealed by immunological analysis with anti-3006 serum, possessing an additional OMP antigen of molecular weight 28000 (Fig. 4c).

Similar analysis of OMC from strains 2396 and 3459, designated serotype $2 \mathrm{c}$, revealed one type-specific OMP antigen, of molecular weight 41500 (Fig. $4 d$ ).

The detailed chemical nature of these serotype 2 subtypes is given in Table 1 .

\section{Distribution of the characterized serotypes among patients and carriers}

Of 215 strains of meningococci, isolated either from cases of meningococcal disease or from healthy carriers, 98 could be assigned, by immunodiffusion of $\mathrm{OMC}$, into one of the 11 serotypes. Of these 98 strains, 75 belonged to either serotype $2 \mathrm{a}$ ( 35 strains), $2 \mathrm{~b}$ (24 strains) or 2c (16 strains). The majority of serotype 2a strains fell into serogroup W-135, particularly those isolated from patients, the other serotype $2 \mathrm{a}$ strains belonging to serogroups $\mathrm{B}, \mathrm{C}$ and $Y$. Of the serotype $2 \mathrm{~b}$ strains, which were all amongst the meningococci isolated from patients, $22 / 24$ belonged to serogroup B. All the serotype $2 \mathrm{c}$ strains were associated with serogroup $\mathrm{Y}$. Of the other serotypes represented in this series, serotype 8 was predominant (Table 2).

\section{DISCUSSION}

The analysis of the OMC of nine meningococcal serotypes described by Frasch et al. (1976) by both immunodiffusion and SGIP enabled the chemical nature of the principal antigen(s) to be identified. In addition, two new serotypes, immunologically related to serotype 2 (Frasch et al., 1976), were recognized.

The SGIP technique proved to be the more sensitive of the two methods of analysis since antisera exhibiting type-specific activity in immunodiffusion analysis gave cross-reactions when tested by the SGIP technique. However, the extensive absorption of such antisera with cross-reacting strains enabled antisera specific in the SGIP test to be developed. Using this approach, it was possible to demonstrate which proteins and lipopolysaccharides (LPS) within the outer membrane were the antigens responsible for serotype-specificity and that these antigens were stable when prepared as described, since different preparations gave identical results. 
Frasch \& Gotschlich (1974) showed the serotype 2 antigen, here renamed serotype 2a, to be an OMP of molecular weight 41000 . Zollinger \& Mandrell (1977) found that the prototype strains M981 (serotype 4), M992 (serotype 5) and M978 (serotype 8) stimulated the production of LPS-specific antibodies in the solid-phase radioimmunoassay. Both these findings have been confirmed by us.

The serotypes $2 \mathrm{a}, 2 \mathrm{~b}$ and $2 \mathrm{c}$ formed the largest group of typable strains isolated from patients and, with the exception of serotype $2 \mathrm{~b}$, from carriers. Unlike Frasch \& Chapman (1973), Jones \& Tobin (1976) and Frasch (1977) we found no serotype 2a strains amongst those serogroup B meningococci isolated from cases of meningitis although, as also noted by Frasch \& Chapman (1973), a few were found amongst carrier strains. However, just over half of the serogroup B strains isolated from cases of meningitis proved to be serotype $2 b$. This discrepancy may well be due to the recognition, reported here for the first time, of other serotype 2 strains. * The remainder of the serotype $2 b$ strains, two in number, belonged to serogroup C. We, like Gold et al. (1971) and Munford et al. (1975), found some serotype 2a strains amongst the serogroup $C$ strains. The vast majority of serogroup W-135 strains contained the serotype $2 \mathrm{a}$ antigen, the incidence being greater amongst the strains isolated from patients. Like Frasch \& Friedman (1977) we found serotype 2a to be present amongst serogroup $\mathrm{Y}$ strains isolated from patients in the U.S.A. Interestingly, this serotype was absent from serogroup $Y$ strains isolated in the Netherlands. Moreover, serotype 2c strains were more common amongst serogroup $Y$ strains isolated in the U.S.A. than amongst those isolated in the Netherlands. These two findings might explain the higher attack rate of serogroup Y in the U.S.A. compared with Europe (Meningococcal Disease Surveillance Group, 1976; Communicable Disease Surveillance Centre, 1978). Investigation of the geographical distribution of serotypes $2 a, 2 b$ and $2 c$ seems desirable.

On the basis of the findings discussed, serotypes $2 a, 2 b$ and $2 c$ could be regarded as meningococcal virulence factors. It appears from our immunochemical investigations that a serotype $2 \mathrm{a}$ vaccine, although cross-reactive with serotypes $2 \mathrm{~b}$ and $2 \mathrm{c}$, will not be the most suitable for protection against serogroup B meningitis (Frasch, 1977; Zollinger et al., 1978). Perhaps, the serotype $2 b$ antigen should be chosen for this purpose.

This investigation reveals that meningococcal OMP contain common immunodeterminants in addition to strain-specific components. Meningococci may contain more than one specific OMP, e.g. strains B16B6 (serotype 2a), M1011 (serotype 2a), S3032 (serotype 12) and 3006 (serotype $2 b$ ). With the exception of serotypes $2 a, 2 b$ and $2 c$, great heterogeneity exists amongst these strain-specific components. This suggests that OMP serotyping will be very broad and not suitable for the serological classification of meningococci. On the other hand, the heterogeneity amongst meningococcal OMP may be of assistance, in association with SDS-PAGE, in the elucidation of subtle or difficult epidemiological problems.

The ability of meningococcal LPS to induce type-specific antisera indicates the possibility for an LPS serotyping scheme. Possibly, the LPS show less heterogeneity than the OMP. Experiments to determine the value of meningococcal LPS for serological classification purposes have been carried out by Zollinger \& Mandrell (1977). An LPS serotyping system is under development in our own laboratory.

* Note added in proof. The prototype serotype $2 \mathrm{~b}$ and 2c strains 2996, 3006, 2396 and 3459 were protein serotyped and belonged to Frasch's serotype 2 by the bactericidal method and by co-agglutination (D. M. Jones, personal communication).

We are grateful to Dr C. E. Frasch for generously providing the prototype strains and some serotype reference antisera and to Dr H. A. Feldman for kindly sending us 20 serogroup Y strains from cases in the U.S.A. We thank Mrs S. S. Speet-Weinreich for her assistance with translating this article into English. This investigation was supported by a grant from the Preventie Fonds, researchnr. 28-310. 


\section{REFERENCES}

Bhattacharjee, A. K., Jennings, H. J. \& Kenny, C. P. (1978). Structural elucidation of the 3deoxy-D-manno-octulosonic acid containing meningococcal 29-e capsular polysaccharide antigen using carbon-13 nuclear magnetic resonance. Biochemistry 17, 645-651.

BRANHAM, S. E. (1953). Serological relationships among meningococci. Bacteriological Reviews 17 $175-188$.

Communicable Disease Surveillance Centre (1978). Acute meningococcal infections, 1977. British Medical Journal 1, 1560.

Evans, J. R., Artenstein, M. S. \& Hunter, D. H. (1968). Prevalence of meningococcal serogroups and description of three new groups. American Journal of Epidemiology 87, 643-646.

Frasch, C. E. (1977). Role of protein serotype antigens in protection against disease due to Neisseria meningitidis. Journal of Infectious Diseases 136S, S84-S90.

Frasch, C. E. \& Chapman, S. S. (1972a). Classification of Neisseria meningitidis group B into distinct serotypes. I. Serological typing by a microbactericidal method. Infection and Immunity 5, 98-102.

Frasch, C. E. \& Chapman, S. S. (1972b). Classification of Neisseria meningitidis group B into distinct serotypes. II. Extraction of type-specific antigens for serotyping by precipitin techniques. Infection and Immunity 6, 127-133.

Frasch, C. E. \& Chapman, S. S. (1973). Classification of Neisseria meningitidis into distinct serotypes. III. Application of a new bactericidalinhibition technique to distribution of serotypes among cases and carriers. Journal of Infectious Diseases 127, 149-154.

Frasch, C. E. \& Friedman, G. L. (1977). Identification d'un serotype meningococcique associé à la maladie et commun aux meningocoques des serogroupes $\mathrm{B}, \mathrm{C}, \mathrm{Y}$ et $\mathbf{1 3 5} \mathrm{W}$. Médecine tropicale 37, 155-159.

Frasch, C. E. \& Gotschlich, E. C. (1974). An outer membrane protein of Neisseria meningitidis group B responsible for serotype specificity. Journal of Experimental Medicine 140, 87-104.

Frasch, C. E., McNelis, R. M. \& Gotschlich, E. C. (1976). Strain-specific variation in the protein and lipopolysaccharide composition of the group B meningococcal outer membrane. Journal of Bacteriology 127, 973-981.

Gold, R. \& WyLE, F. A. (1970). New classification of Neisseria meningitidis by means of hactericidal reactions. Infection and Immunity 1, 479-484.

Gold, R., Winkelhake, J. L., Mars, R. S. \& Artenstein, M.S. (1971). Identification of an epidemic strain of group C Neisseria meningitidis by bactericidal serotyping. Journal of Infectious Diseases 124, 593-597.
Jennings, H. J., Bhattacharjee, A. K., Bundle, D. R., Kenny, C. P., Martin, A. \& SMith, I. C. P. (1977). Structures of the capsular polysaccharides of Neisseria meningitidis as determined by ${ }^{13} \mathrm{C}$-nuclear magnetic resonance spectroscopy. Journal of Infectious Diseases 136S, S71-S77.

JoNES, D. M. \& ToBIN, B. M. (1976). Serotypes of group B meningococci. Journal of Clinical Pathology 29, 746-748.

MANDRell, R. E. \& Zollinger, W. D. (1977). Lipopolysaccharide serotyping of Neisseria meningitidis by hemagglutination inhibition. Infection and Immunity 16, 471-475.

Meningococcal Disease Surveillance Group (1976). Analysis of endemic meningococcal disease by serogroup and evaluation of chemoprophylaxis. Journal of Infectious Diseases 134, 201-204.

Munford, R. S., Patton, C. M. \& Gorman, G. W. (1975). Epidemiological studies of serotype antigens common to groups $\mathrm{B}$ and $\mathrm{C}$ Neisseria meningitidis. Journal of Infectious Diseases 131, 286-290.

Poolman, J. T., Hopman, C. T. P. \& Zanen, H. C. (1978). Immunochemical characterization of outer membrane complexes from Neisseria meningitidis by the SDS-polyacrylamide-gel-electrophoresis-immuno-peroxidase technique (SGIP). FEMS Microbiology Letters 4, 245-248.

RaAmsdonk, W. v., Pool, C. W. \& Heyting, C. (1977). Detection of antigens and antibodies by an immuno-peroxidase method applied on thin longitudinal sections of SDS-polyacrylamide gels. Journal of Immunological Methods 17, 337-348.

Slaterus, K. W. (1961). Serological typing of meningococci by means of micro-precipitation. Antonie van Leeuwenhoek 27, 305-315.

Zollinger, W. D. \& MANDRell, R. E. (1977). Outer-membrane protein and lipopolysaccharide serotyping of Neisseria meningitidis by inhibition of a solid-phase radioimmunoassay. Infection and Immunity 18, 424-433.

Zollinger, W. D., Kasper, D. L., Veltri, B. J. \& ARTENSTEIN, M. S. (1972). Isolation and characterization of a native cell wall complex from Neisseria meningitidis. Infection and Immunity $\mathbf{6}$, 835-851.

Zollinger, W. D., Pennington, C. L. \& Artenstein, M. S. (1974). Human antibody response to three meningococcal outer membrane antigens: comparison by specific hemagglutination assays. Infection and Immunity 10, 975-984.

Zollinger, W. D., Mandrell, R. E., Altieri, P., Berman, S., Lowenthal, J. \& Artenstein, M. S. (1978). Safety and immunogenicity of a Neisseria meningitidis type 2 protein vaccine in animals and humans. Journal of Infectious Diseases 137, 728-739. 